\title{
A Rare Complication Observed during Percutaneous Nephrolithotomy: Foreign Body Migration from the Right Kidney to the Left Lung
}

\author{
Mehmet Kaba $^{1}$ Necip Pirinççi ${ }^{1} \quad$ Sultan Kaba ${ }^{2} \quad$ Ufuk Çobanoğlu ${ }^{3}$ Recep Eryılmaz ${ }^{1}$ Hüseyin Eren ${ }^{1}$ \\ ${ }^{1}$ Department of Urology, Faculty of Medicine, Yuzuncu Yıl University, \\ Van, Turkey \\ 2 Department of Pediatric, Faculty of Medicine, Yuzuncu Yıl University, \\ Van, Turkey \\ ${ }^{3}$ Department of Thoracic Surgery, Faculty of Medicine, Yuzuncu Yıl \\ University, Van, Turkey \\ Eur J Pediatr Surg Rep 2015;3:15-17. \\ Faculty of Medicine, Yuzuncu Yıl University, Van 65100, Turkey \\ (e-mail: mehmetkaba@yahoo.com).
}

\author{
Abstract \\ Keywords \\ - foreign body \\ - migration \\ - percutaneous \\ nephrolithotomy
}

Percutaneous nephrolithotomy (PNL) is the first-line treatment in large, multiple stones and lower calyceal stones. Majority of complications associated with PNL are minor and clinically insignificant. It was seen that distal piece $(2 \mathrm{~cm}$ in size) of ureter catheter observed at pelvis was found at the parenchyma of left lung on the perioperative fluoroscopy in the patient undergoing PNL for right kidney stone. We presented this complication to stress that a foreign body can pass into circulation presumably through venous injury and can migrate to the lung.

\section{Introduction}

There are many therapeutic options for the management of urinary stones. In the management of upper urinary tract stone disease, the method of dilating urinary tract via percutaneous renal puncture to extract urinary calculi was first introduced in 1976 by Fernström and Johansson. ${ }^{1}$ Percutaneous nephrolithotomy (PNL) became a first-line treatment for most patients with large-volume renal and staghorn stones after advances in equipment and surgical techniques. ${ }^{2}$

PNL was first introduced in pediatric population in 1985. Initially, there were concerns regarding the use of PNL for pediatric stone disease, but it is at present a routine surgical method and has replaced open surgery. ${ }^{3}$ However, serious complications can occur during and after PNL, although it has high success rates in the treatment of pediatric and adult stone diseases.

Here, we presented a 15-year-old girl in whom we thought that a right pelvic foreign body migrated to left lung via venous system; as such no complication has been reported in the literature so far.

received

December 24, 2013 accepted after revision January 18, 2014 published online August 4, 2014

\section{Case Report}

A 15-year-old girl was admitted to the hospital with a diagnosis of right kidney for surgical intervention. On physical examination, only flank pain was noted in the right kidney. The patient had no abnormal finding in her history. Urinary system sonography revealed enlargement with dilated renal pelvis and a stone $(\sim 2 \mathrm{~cm}$ in diameter) in right kidney. On a computed tomography (CT) scan, a stone $(20 \times 10 \mathrm{~mm}$ in size $)$ was detected at the right renal pelvis ( - Fig. 1). Right pelvicalyceal system was found to be dilated (grade II hydronephrosis). Previous extracorporeal shock wave lithotripsy intervention was failed.

Before surgery, a $4 \mathrm{~F}$ ureteral catheter was inserted through the orifice of right ureter by cystoscopy guidance to provide access for performing perioperative retrograde pyelogram during surgery. Then, the patient was placed in a prone position and an access to kidney was provided via lower calyx. Renal pelvis was exposed by using dilators. Calculus at renal pelvis was fragmented by pneumatic lithotripter. A distal part of the catheter $(\sim 2 \mathrm{~cm}$ in size $)$ was detached
License terms

Stuttgart - New York

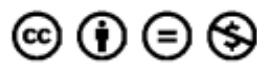




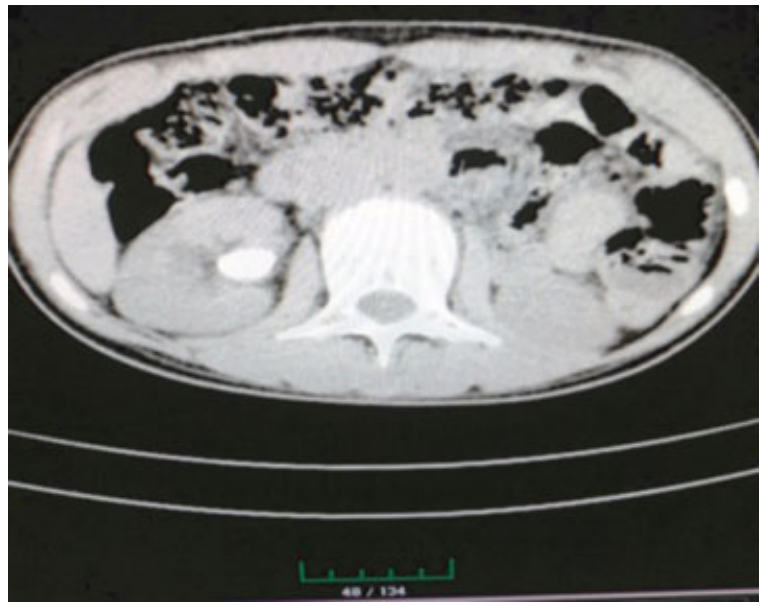

Fig. 1 A noncontrast computed tomography scan showing a 2-cm stone in the right renal pelvis.

during extraction of the fragmented calculi by stone forceps. When pelvicalyceal system was explored for foreign body after the removal of fragmented calculi, it failed to find the foreign body which was previously observed on fluoroscopy. By fluoroscopy assistance, the foreign body was located within pulmonary parenchyma at left. The operation was completed after consulting with thoracic surgery. Preoperative hemoglobin and hematocrit levels were $10.3 \mathrm{~g} / \mathrm{dL}$ and $32.4 \%$, respectively. On day 1 after surgery, hemoglobin and hematocrit levels were measured as $9.7 \mathrm{~g} / \mathrm{dL}$ and $30.6 \%$, respectively. No blood transfusion was needed. On day 2 , a dilatation was observed at right pelvicalyceal system (grade II hydronephrosis) on the sonography. In addition, sonography showed percutaneous nephrostomy tube, which entered into the right kidney from lower pole and ended at the renal pelvis. On the postoperative CT scan, linear atelectasis was observed at lateral region of inferior lobe in left lung. Also, there were two linear hyperdense areas with close vicinity (foreign body) at lingua of left lung (- Fig. 2). The patient was operated by thoracic surgery department on day 8 and a rest catheter ( $2 \mathrm{~cm}$ in size) was removed from pulmonary parenchyma via a left video thorascopic surgery (-Fig. 3). A

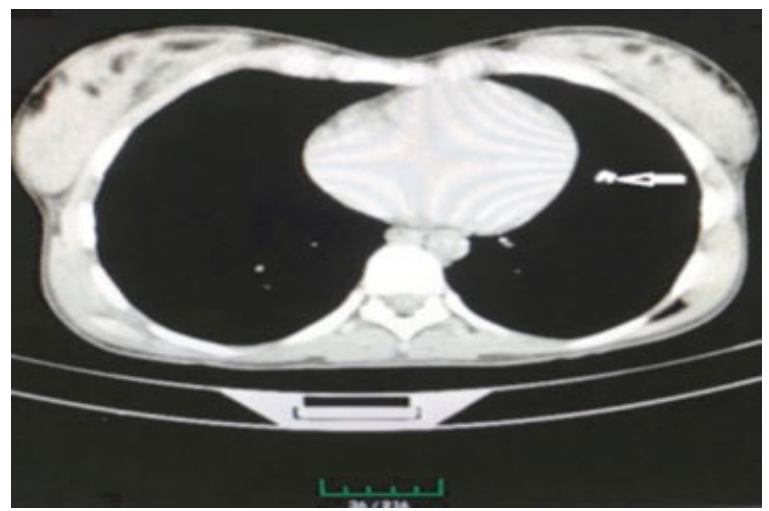

Fig. 2 A computed tomography scan showing two linear hyperdense areas in close vicinity on the left pulmonary parenchyma.

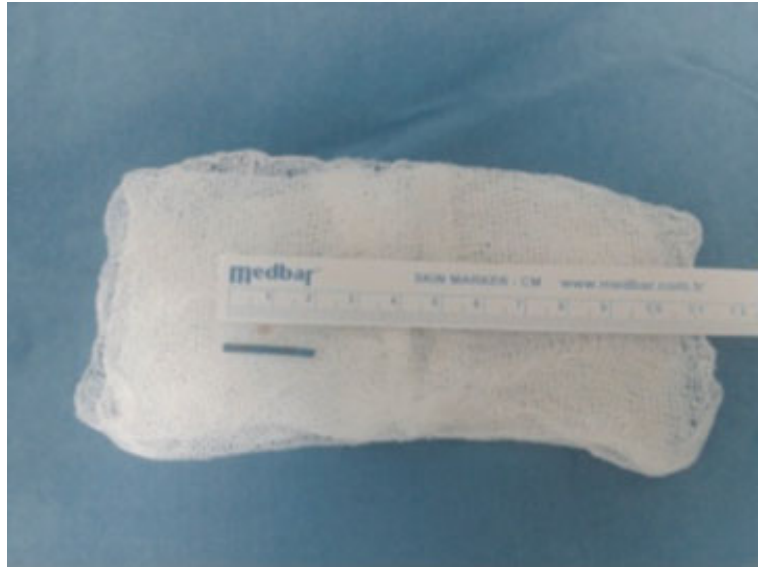

Fig. 3 A piece of the stent removed from the left pulmonary parenchyma via a video thorascopic surgery.

thorascopic surgical approach was preferred instead of the endoluminal approach for foreign bodies that extravasated from the vascular tissue into the lung parenchyma. The patient was discharged on day 6 after second operation, as she had no complication during the postoperative follow-up.

\section{Discussion}

Although PNL is a surgical method with high success rates, it should be kept in mind that complications can occur during and after the procedure. The most common complications include extravasation (7.2\%), bleeding requiring blood transfusion (11.2-17.5\%), and fever (21-32.1\%) while less common complications are septicemia $(0.3-4.7 \%)$, colon injury $(0.2-$ $4.8 \%$ ), and pleural lacerations $(0-3.1 \%) .{ }^{4}$ To the best of our knowledge, migration of foreign body to lung through venous route has yet not been reported in the literature so far.

In percutaneous procedures, terms of minor and major complications have not been standardized yet; thus, it is difficult to make a comparison. Data regarding complications should be obtained in a standardized manner to allow comparison and make a reproducible quality assessment. In this context, it is reasonable to stratify complications based on timing (intraoperative or postoperative). The Dindo-modified Clavien system is a useful tool, which was validated in 6,336 patients for quality assessment in surgery. ${ }^{5}$ Intrathoracic complications included pneumothorax, hydrothorax, hemothorax, and urinothorax. ${ }^{6}$ Although, there are a few animal studies addressing intrarenal or pyelovenous reflux, resulting from elevated intrarenal pressure in percutaneous procedures, postoperative fever is generally attributed to bacterial reflux and endotoxin or bacterial absorption. ${ }^{7,8}$ In a previous study, fluid absorption was evaluated in 148 patients by using estimations of breath ethanol concentration. ${ }^{9}$ In that study, it was found that volume of fluid absorbed increased with the amount of irrigation fluids, pelvicalyceal perforation, bleeding, and duration of procedure but not related to the number of tracts. There was no clinical and laboratory evidence of electrolyte imbalance, but fluid 
absorption was evident in all patients. Authors reported that staged nephrostomy through mature dilated tract was helpful in reducing the amount of fluid absorbed. ${ }^{9}$

While extracting fragments of renal calculus by forceps, it can not only cause damage in renal pelvis but also rupture of ureteral catheter and stripping of sensor guides, if not used meticulously. Interestingly, we think that distal part of ruptured ureteral catheter migrated to the right heart through an opening at one of the intrarenal branches of right renal vein and, in turn, left lung through right pulmonary artery. The catheter fragment was removed via a left video-assisted thoracoscopic surgery by thorax surgery department as thorax surgeons recommended that the removal of foreign body would be indicated due to age of patient and potential to cause further complication by migrating out of the lung

We aimed to stress that one should keep in mind that foreign bodies (catheter pieces, stone fragments) can migrate into the lung through circulation even in the lack of perioperative findings, which indicates vascular injury, such as bleeding, in patients undergoing PNL during intraoperative monitoring or postoperative follow-up.

Conflict of Interest

None.

\section{References}

1 Fernström I, Johansson B. Percutaneous pyelolithotomy. A new extraction technique. Scand J Urol Nephrol 1976;10(3):257-259

2 Preminger GM, Assimos DG, Lingeman JE, Nakada SY, Pearle MS, Wolf JS Jr; AUA Nephrolithiasis Guideline Panel). Chapter 1: AUA guideline on management of staghorn calculi: diagnosis and treatment recommendations. J Urol 2005;173(6):1991-2000

3 Rizvi SA, Naqvi SA, Hussain Z, et al. The management of stone disease. BJU Int 2002;89(Suppl 1):62-68

4 Michel MS, Trojan L, Rassweiler JJ. Complications in percutaneous nephrolithotomy. Eur Urol 2007;51(4):899-906, discussion 906

5 Dindo D, Demartines N, Clavien PA. Classification of surgical complications: a new proposal with evaluation in a cohort of 6336 patients and results of a survey. Ann Surg 2004;240(2): 205-213

6 Munver R, Delvecchio FC, Newman GE, Preminger GM. Critical analysis of supracostal access for percutaneous renal surgery. J Urol 2001;166(4):1242-1246

7 Nagele U, Horstmann M, Sievert KD, et al. A newly designed amplatz sheath decreases intrapelvic irrigation pressure during mini-percutaneous nephrolitholapaxy: an in-vitro pressure-measurement and microscopic study. J Endourol 2007;21(9):1113-1116

8 Zhong W, Zeng G, Wu K, Li X, Chen W, Yang H. Does a smaller tract in percutaneous nephrolithotomy contribute to high renal pelvic pressure and postoperative fever? J Endourol 2008;22(9): 2147-2151

9 Kukreja RA, Desai MR, Sabnis RB, Patel SH. Fluid absorption during percutaneous nephrolithotomy: does it matter? J Endourol 2002; 16(4):221-224 